\title{
Transcriptome-based molecular systematics: Rhodnius montenegrensis (Triatominae) and its position within the Rhodnius prolixus-Rhodnius robustus cryptic-species complex
}

\author{
Raíssa N. Brito ${ }^{1}$, Juliana A. Geraldo ${ }^{2,3}$, Fernando A. Monteiro ${ }^{4}$, Cristiano Lazoski ${ }^{5}$ Rita C. M. Souza ${ }^{1}$ \\ and Fernando Abad-Franch ${ }^{1,6^{*}}$
}

\begin{abstract}
Background: Rhodnius montenegrensis (Triatominae), a potential vector of Chagas disease, was described after $R$. robustus-like bugs from southwestern Amazonia. Mitochondrial cytb sequence near-identity with sympatric $R$. robustus (genotype II) raised doubts about the taxonomic status of $R$. montenegrensis, but comparative studies have reported fairly clear morphological and genetic differences between R. montenegrensis and laboratory stocks identified as $R$. robustus. Here, we use a transcriptome-based approach to investigate this apparent paradox.

Results: We retrieved publicly-available transcriptome sequence-reads from $R$. montenegrensis and from the $R$. robustus stocks used as the taxonomic benchmark in comparative studies. We (i) aligned transcriptome sequence-reads to mitochondrial (cytb) and nuclear (ITS2, D2-28S and AmpG) query sequences (47 overall) from members of the $R$. prolixus-R. robustus cryptic-species complex and related taxa; (ii) computed breadth- and depth-coverage for the 259 consensus sequences generated by these alignments; and, for each locus, (iii) appraised query sequences and fullbreadth-coverage consensus sequences in terms of nucleotide-sequence polymorphism and phylogenetic relations. We found evidence confirming that $R$. montenegrensis and $R$. robustus genotype II are genetically indistinguishable and, hence, implying that they are, in all likelihood, the same species. Furthermore, we found compelling genetic evidence that the benchmark' $R$. robustus' stocks used in R. montenegrensis description and in later transcriptome-based comparisons are in fact $R$. prolixus, although likely mixed to some degree with $R$. robustus (probably genotype II, a.k.a. R. montenegrensis).
\end{abstract}

Conclusions: We illustrate how public-domain genetic/transcriptomic data can help address challenging issues in disease-vector systematics. In our case-study, taxonomic confusion apparently stemmed from the misinterpretation of sequence-data analyses and misidentification of taxonomic-benchmark stocks. More generally, and together with previous reports of mixed and/or misidentified Rhodnius spp. laboratory colonies, our results call into question the conclusions of many studies (on morphology, genetics, physiology, behavior, bionomics or interactions with microorganisms including trypanosomes) based on non-genotyped 'R. prolixus' or 'R. robustus' stocks. Correct species identifcation is a prerequisite for investigating the factors that underlie the physiological, behavioral or ecological differences between primary domestic vectors of Chagas disease, such as $R$. prolixus, and their sylvatic, medically less-relevant relatives such as $R$. robustus (s.l.) including $R$. montenegrensis.

Keywords: Rhodnius, Triatominae, Molecular systematics, Transcriptomics

\footnotetext{
${ }^{*}$ Correspondence: abadfr@yahoo.com

${ }^{1}$ Grupo Triatomíneos, Instituto René Rachou, Fiocruz Minas Gerais,

Fundação Oswaldo Cruz (Fiocruz), Belo Horizonte, Brazil

Full list of author information is available at the end of the article
} 


\section{Background}

Rhodnius prolixus (Triatominae) is a primary vector of Chagas disease across northern South America, where infestation of rural houses by this species is common $[1,2]$; it belongs to a group of closely-related taxa with nearly identical morphologies, i.e. the ' $R$. prolixus $-R$. robustus cryptic-species complex' [3-6]. Except for $R$. prolixus, the species in this complex ( $R$. robustus (s.l.) [5, 6], $R$. montenegrensis [7] and R. marabaensis [8]) do not infest houses and have relatively little medical relevance [4, 9-11]. Why just one species within this group of close-kin bugs has the ability to stably infest houses is still unclear $[6,9,11]$. At least in part, this knowledge gap stems from the taxonomic uncertainty inherent to studying cryptic taxa. Thus, even though molecular systematics has contributed substantially to clarify the composition of the $R$. prolixus $-R$. robustus complex and the relations among its members, some controversies remain [3-6]. Using $R$. montenegrensis as a case-study, here we describe an approach that combines publicdomain genetic/transcriptomic data and bioinformatics to address such controversies.

Rhodnius montenegrensis was described in 2012 by researchers of the Universidade Estadual Paulista 'Júlio de Mesquita Filho' (UNESP), Brazil, based on bugs resembling $R$. robustus [7]. The material used in this description came from UNESP colony 'CTA 88', which was founded with eight bugs collected in 2008 from Attalea palms in the southwestern Brazilian Amazon [7]. These bugs were compared to benchmark material identified as $R$. robustus from four laboratory colonies ('CTA 83' to 'CTA 86') founded with bugs collected in Peru and kept at UNESP since the early 1970s [7]. The original description included a 369-bp DNA sequence of $R$. montenegrensis' mitochondrial cytochrome $\mathrm{b}$ (cytb) gene (GenBank ID: KR072682.1); a phylogenetic analysis recovered KR072682.1 as very closely related to a sequence of undisclosed origin or GenBank ID but labeled as ' $R$. robustus' (see figure 15 in [7]). In addition, the endonuclease Bst $\mathrm{UI}$ did not cleave nuclear rDNA 5.8S/ITS2 amplicons from putative $R$. montenegrensis, but cleaved at one site amplicons from the benchmark $R$. robustus colony bugs, thus implying that the $5.8 \mathrm{~S} /$ ITS2 sequences of $R$. montenegrensis and $R$. robustus differ by at least one base at the enzyme's restriction site [7]. The authors concluded that, although closely related, $R$. montenegrensis and $R$. robustus are morphologically and genetically distinct [7]. These findings received further support from a comparativetranscriptomics study showing that bugs identified as $R$. montenegrensis and bugs from UNESP's $R$. robustus colony 'CTA 85', had “... a substantial quantity of fixed interspecific polymorphisms ..."; this was interpreted as "... suggest[ing] a high degree of genetic divergence between the two species [that] likely corroborates the species status of $R$. montenegrensis" ([12], Abstract; see also [13] for details).

The striking similarity of $R$. montenegrensis and $R$. robustus cytb sequences was recently confirmed by a broader analysis [6] showing that $R$. montenegrensis' KR072682.1 is nearly identical to $c y t b$ sequences from $R$. robustus genotype II, one of the $R$. robustus cryptic taxa identified by Monteiro et al. [5] in the early 2000s. While these results clearly suggest that $R$. montenegrensis is "... part of the variability of $R$. robustus II ..." (caption of figure $2 \mathrm{~A}$ in [6]), they raise the question of why morphology [7] and transcriptomics [12, 13] both discriminate $R$. montenegrensis from $R$. robustus bugs of Peruvian origin. This is even more intriguing when one considers that $R$. robustus II is the only $R$. robustus lineage known to occur in western-southwestern Amazonia $[5,6,14-16] ; R$. robustus material from Peru, then, is expected to belong in genotype II and, hence, to be indistinguishable from $R$. montenegrensis.

Recently, Monteiro and colleagues [6] suggested a possible explanation for these apparently contradictory findings. They observed (i) that the members of the $R$. prolixus $-R$. robustus species complex all have virtually identical phenotypes [1-4]; (ii) that several speciespairs within the complex are inter-fertile [17]; (iii) that there is evidence that many laboratory colonies of bugs identified as either $R$. prolixus or $R$. robustus (s.l.) are mixed/contaminated or wrongly labeled (see SI Appendix of [18]); and (iv) that cytb sequences of bugs from colonies labeled as ' $R$. robustus' from 'Lima, Peru' match $R$. prolixus sequences [19]. These observations suggest that the Peruvian $R$. robustus colonies kept at UNESP may have become contaminated with non- $R$. robustus material, with the main suspect being $R$. prolixus [6]. This hypothesis predicts that bugs drawn from the Peruvian ' $R$. robustus' colonies at UNESP will have $R$. prolixus genetic material, perhaps mixed to some degree with $R$. robustus (likely genotype II). In contrast, bugs from the younger (and hence less likely to have become contaminated) $R$. montenegrensis colonies should be genetically indistinguishable from $R$. robustus II. Here, we use publicly-available transcriptome data derived from UNESP Rhodnius spp. colonies to test these two predictions. More generally, we present a methodological approach (Fig. 1) that leverages publicdomain information from traditional and next-generation sequencing projects to investigate the molecular systematics of cryptic species in the face of taxonomic confusion. 


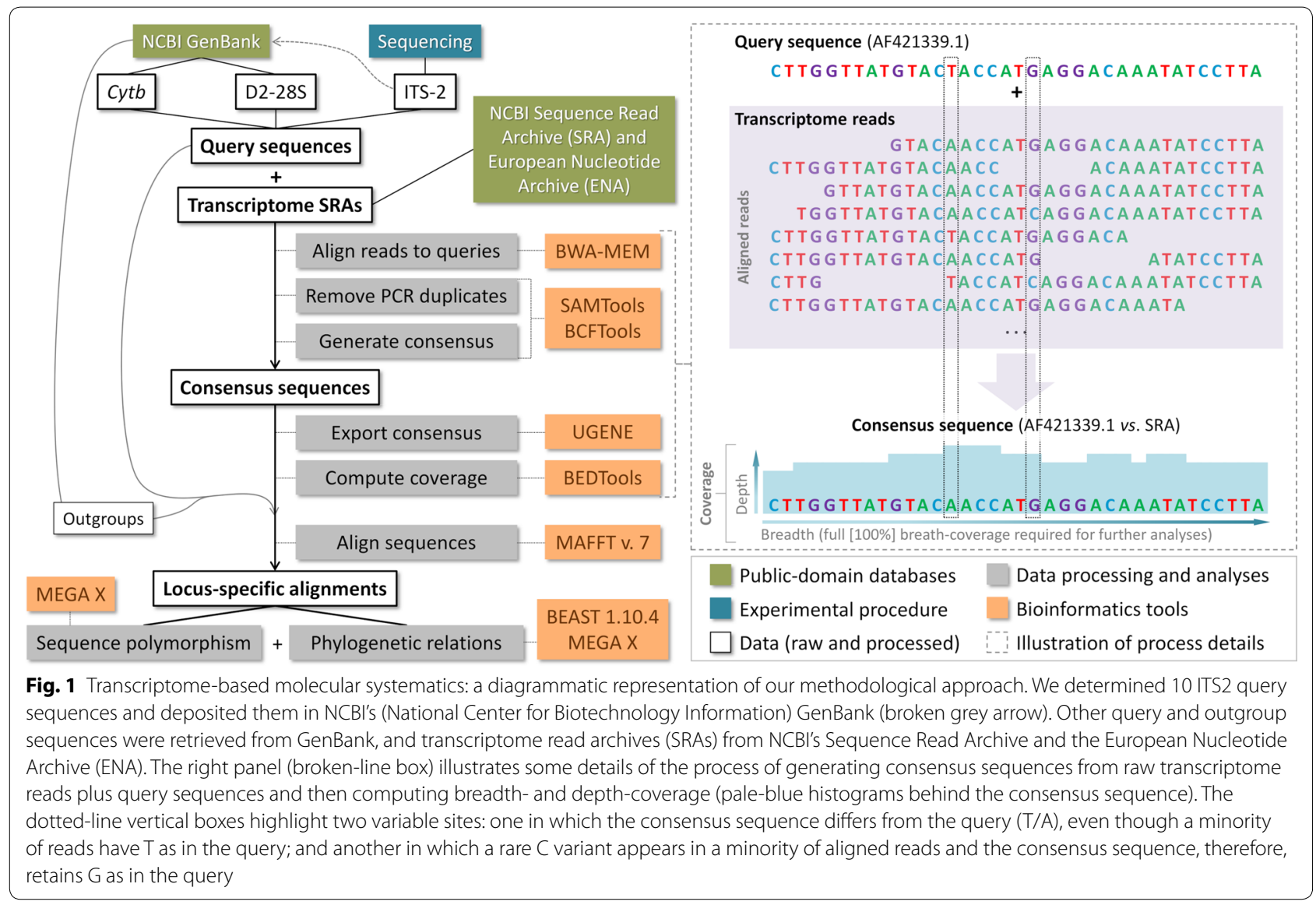

Table 1 Transcriptome raw data (sequence read archives) used in this study

\begin{tabular}{|c|c|c|c|c|c|c|c|}
\hline Putative species & SRA & ENA run & Platform ${ }^{a}$ & Colony & Material & Notes & Reference \\
\hline \multirow[t]{2}{*}{ Rhodnius montenegrensis } & SRX1996481 & SRR3995415 & HiSeq 2500 & UNESP 'CTA 88' & Heads & Pool of 7 males & {$[12,13]$} \\
\hline & SRX1996482 & SRR3995416 & HiSeq 2500 & UNESP 'CTA 88' & Heads & Pool of 6 males & {$[12,13]$} \\
\hline \multirow[t]{4}{*}{ Rhodnius robustus } & SRX1996483 & SRR3995417 & HiSeq 2500 & UNESP 'CTA 85' & Heads & Pool of 6 males & {$[12,13]$} \\
\hline & SRX1996484 & SRR3995418 & HiSeq 2500 & UNESP 'CTA 85' & Heads & Pool of 6 males & {$[12,13]$} \\
\hline & ERX1387159 & ERR1315266 & HiSeq 2000 & UNESP colony (Peru) & Rostrum + antennae & Pool of 21 females & Unpublished ${ }^{b}$ \\
\hline & ERX1387160 & ERR1315268 & HiSeq 2000 & UNESP colony (Peru) & Rostrum + antennae & Pool of 19 males & Unpublished ${ }^{b}$ \\
\hline
\end{tabular}

a Illumina, $2 \times 100$-bp paired-end reads

b CNRS (Centre National de la Recherche Scientifique, France) sequencing project; see [21]

Abbreviations: SRA, transcriptome access code at the Sequence Read Archive, National Center for Biotechnology Information (NCBI); ENA run, transcriptome run access code at the European Nucleotide Archive, European Molecular Biology Laboratory-European Bioinformatics Institute (EMBL-EBI); UNESP, Universidade Estadual Paulista 'Júlio de Mesquita Filho', Brazil

\section{Methods}

\section{Transcriptome queries}

We used selected DNA sequences from members of the $R$. prolixus $-R$. robustus cryptic-species complex to query publicly-available transcriptome sequence read archives (SRAs) derived from UNESP colonies, namely the $R$. montenegrensis colony and the Peruvian
' $R$. robustus' colonies used as the taxonomic benchmark in the description of $R$. montenegrensis [7] and in later transcriptome-based comparisons [12, 13] (Table 1). We chose three loci that have been widely used to study the systematics and evolution of Rhodnius spp. and other triatomines: the mitochondrial $c y t b[4-6,11,14,16,19]$ plus the nuclear rDNA ITS2 [6, 20] and D2-28S [5]. Cytb 
and D2-28S sequences were retrieved from GenBank, and our ten ITS2 query sequences ( $R$. prolixus and $R$. robustus I to IV; GenBank: MK411269-MK411278) were determined using previously described primers and protocols [20]. Figure 1 presents a summary of our methods, and Additional file 1: Tables S1 and S2 provide details on query sequences including GenBank ID codes.

Figure 1 shows an outline of our methodological approach. We downloaded six public-domain transcriptome SRAs determined by two independent groups at UNESP and the French Centre National de la Recherche Scientifique (CNRS), respectively (see Table 1 and [12, 13, 21]). We aligned transcriptome paired-end reads to our query sequences using the Burrows-Wheeler aligner [22] with the BWA-MEM algorithm and default parameter values except for $-\mathrm{t}=10,-\mathrm{B}=5,-\mathrm{O}=7.7,-\mathrm{L}=6.6$, and $-\mathrm{U}=18$. We stored aligned reads in Binary Alignment Map (BAM) format, removed PCR duplicates with SAMtools markdup [23], and used SAMtools mpileup and BCFtools [23] to generate a consensus sequence for each alignment. We then exported consensus sequences in fasta format using UGENE [24], and computed sequence breadth-coverage and site-specific read depthcoverage using BEDTools [25]. We retained for further analyses consensus sequences with full-breadth-coverage of the query sequence (Fig. 1).

As a quality check for our focal results, we queried SRAs with (i) $c y t b$ and D2-28S sequences from $R$. neglectus and $R$. nasutus, which are relatively close kin to the members of the $R$. prolixus $-R$. robustus species complex [6]; and (ii) sequences of a putative nuclear intron (AmpG) from $R$. prolixus and $R$. robustus I-IV [15]. Our expectation was that these complementary queries would not generate any full-breadth-coverage consensus sequence.

\section{Sequence-polymorphism and phylogenetic analyses}

We used MAFFT v.7 [26] to align, for each locus, query sequences, full-breadth-coverage consensus sequences and selected outgroup GenBank sequences ( $R$. barretti JX273159.1 for cytb, $R$. stali AJ286890.2 for ITS2 and $R$. nasutus AF435856.1 for D2-28S); we made some manual adjustments to the ITS2 and D2-28S alignments (Additional file 2: Alignments S1-S3). Locus-specific alignments were first analyzed in terms of sequence polymorphism using MEGA X [27]. We computed a set of basic sequence diversity and similarity metrics (Additional file 1: Table S1), and examined segregating sites in detail to identify those that might be informative in the context of our research question - e.g., species-diagnostic character states and missense or nonsense mutations in the protein-coding $c y t b$ sequences. We used the bootstrap (1000 pseudo-replicates) to provide estimates of uncertainty for sequence diversity/similarity metrics. We then evaluated phylogenetic relations among the sequences in each alignment using the Bayesian approach implemented in BEAST v.1.10.4 [28]. For each locus, we completed four independent runs with Yule priors for 1,000,000 generations, sampling every 1000 steps and with a 25\% burn-in. We then used FigTree v.1.4.4 (http:// tree.bio.ed.ac.uk/software/figtree) to build maximumcredibility trees with the posterior probability cut-off set at 0.5. We assessed clade support based on posterior probabilities. We also estimated maximum-likelihood (ML) and maximum-parsimony (MP) trees in MEGA $X$ [27]; the methods and results of these complementary analyses can be found in Additional file 3: Figure S1, Additional file 4: Figure S2, Additional file 5: Figure S3 and in the captions thereof. We finally examined the results of our sequence-polymorphism and phylogenetic analyses in the light of sequence depth-coverage, measured as the number of reads that aligned at each nucleotide position (see Fig. 1). In particular, full-breathcoverage consensus sequences with mean depth-coverage $<10$ reads/position were regarded as unreliable [29], and consensus sequences with mean depth-coverage $\geq 10$ reads/position, but with $\geq 15 \%$ of positions supported by $<10$ reads, as dubious (see Additional file 1: Table S1 and Additional file 6: Figure S4).

\section{Results \\ General results}

We aligned sequence reads from six transcriptomes to query sequences representative of all $(c y t b)$ or all but one (ITS2 and D2-28S) known members of the $R$. prolixus- $R$. robustus cryptic-species complex; ITS2 and D2-28S sequences of the little-known $R$. robustus $\mathrm{V}$ are so far unavailable. Overall, our query dataset comprised 47 sequences (Additional file 1: Table S1). Using fairly stringent alignment/filter settings, we generated 61 fullbreadth-coverage and 198 partial-breadth-coverage consensus sequences; no base aligned to the query sequence in 23 of our 282 queries (Additional file 1: Table S1). Depth-coverage was usually high for queries yielding fullbreadth-coverage, but varied substantially across SRAs (Figs. 2, 3, 4, Tables 3, 4; see Additional file 1: Table S1 for breadth- and depth-coverage summary statistics across all queries, and Additional file 6: Figure S4 for fullbreadth-coverage consensus sequences in which depthcoverage was $<10$ reads at one or more positions.

\section{Mitochondrial cytochrome $b$}

The 369-bp $R$. montenegrensis cytb sequence reported in the species' description (KR072682.1) [7] differs at just one to four bases from those of bugs identified as $R$. robustus II collected in Rondônia, Brazil (including 


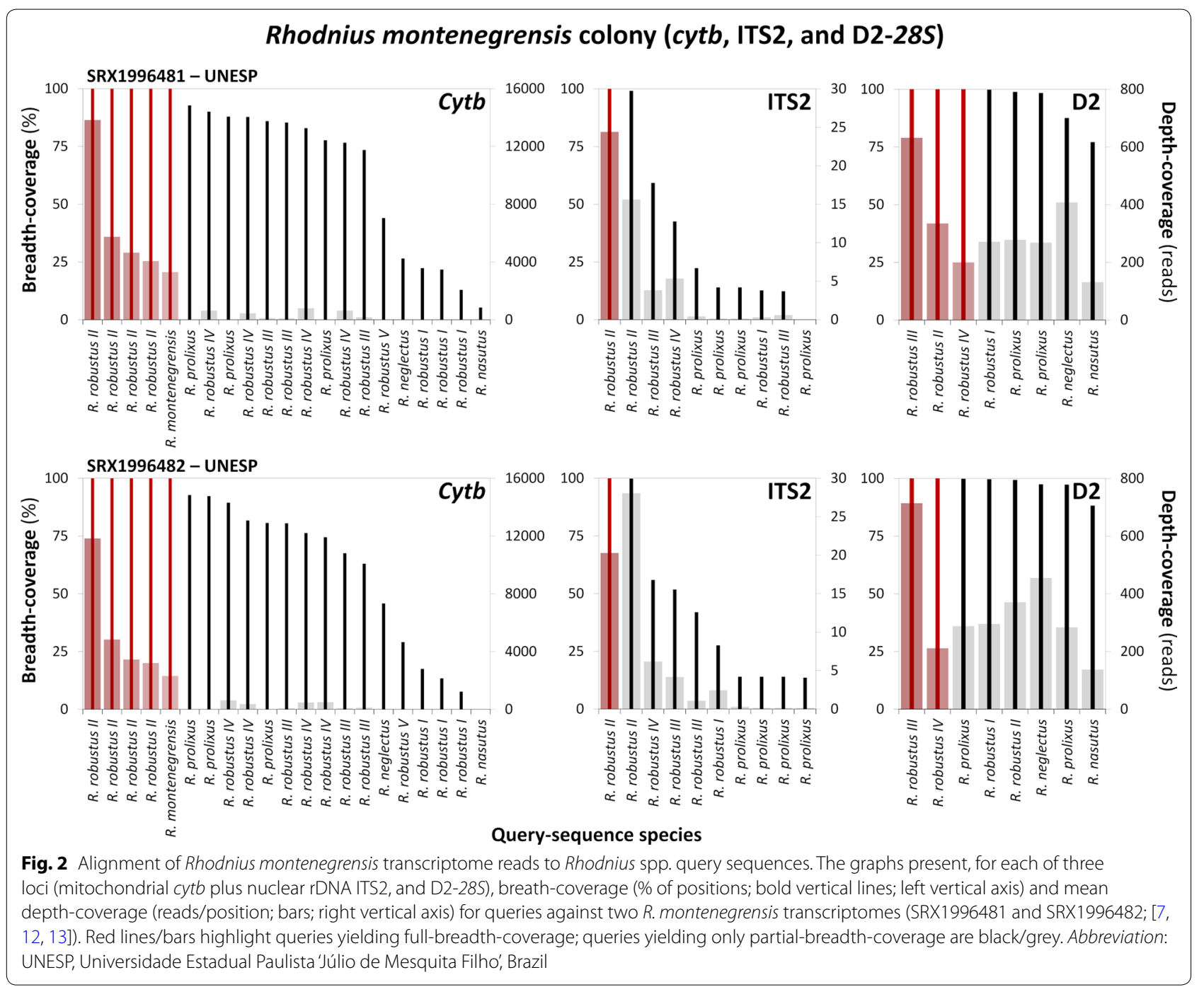

Monte Negro, the type locality of $R$. montenegrensis), and at five bases from that of a bug collected in Napo, Ecuador $[5,14,30]$ (Table 2). We note that all Rhodnius spp. $c y t b$ sequences known to us have A at site 280 in our 663bp alignment; the only exception seems to be EF071583.1 (R. robustus II from Rondônia [30]), which has G, a mutation that yields an Asparagine/Glycine change in the predicted protein (Additional file 2: Alignment S1). We strongly suspect that this first-codon position 'mutation' is a base-call error in EF071583.1. In support of this suspicion, we found that depth-coverage was lowest around position 280 when we aligned $R$. montenegrensis reads (SRAs SRX1996481 and SRX1996482) to query EF071583.1, with values of just $1.4-1.5 \%$ of the mean depth values (Table 3 ).

When we used $R$. montenegrensis' KR072682.1 as the query sequence, $R$. montenegrensis SRAs yielded consensus sequences with full-breadth-coverage and substantial depth-coverage (Table 3, Fig. 2, Additional file 1: Table S1). This was also the case, however, when we aligned the same two SRAs to our three $R$. robustus II $c y t b$ query sequences, with, in addition, consistently improved depth-coverage (Table 3, Fig. 2, Additional file 1: Table S1). Reads from the $R$. montenegrensis SRAs aligned with full-breadth-coverage and substantial depthcoverage to query EF011724.1 (R. robustus II from Monte Negro [14]) (Table 3, Fig. 2, Additional file 1: Table S1). These two consensus sequences were identical, and both differed at a single, second-codon position (A/T, with depth-coverage $>11,000$ reads) from the query sequence. Because all Rhodnius spp. cytb sequences we are aware of, except for $R$. pictipes, have $\mathrm{T}$ at this position (623 in our 663-bp alignment; Additional file 2: Alignment S1), we also suspect a base-call error in EF011724.1. This query sequence, as well as the consensus sequences it generated from the two $R$. montenegrensis SRAs, differed at three 


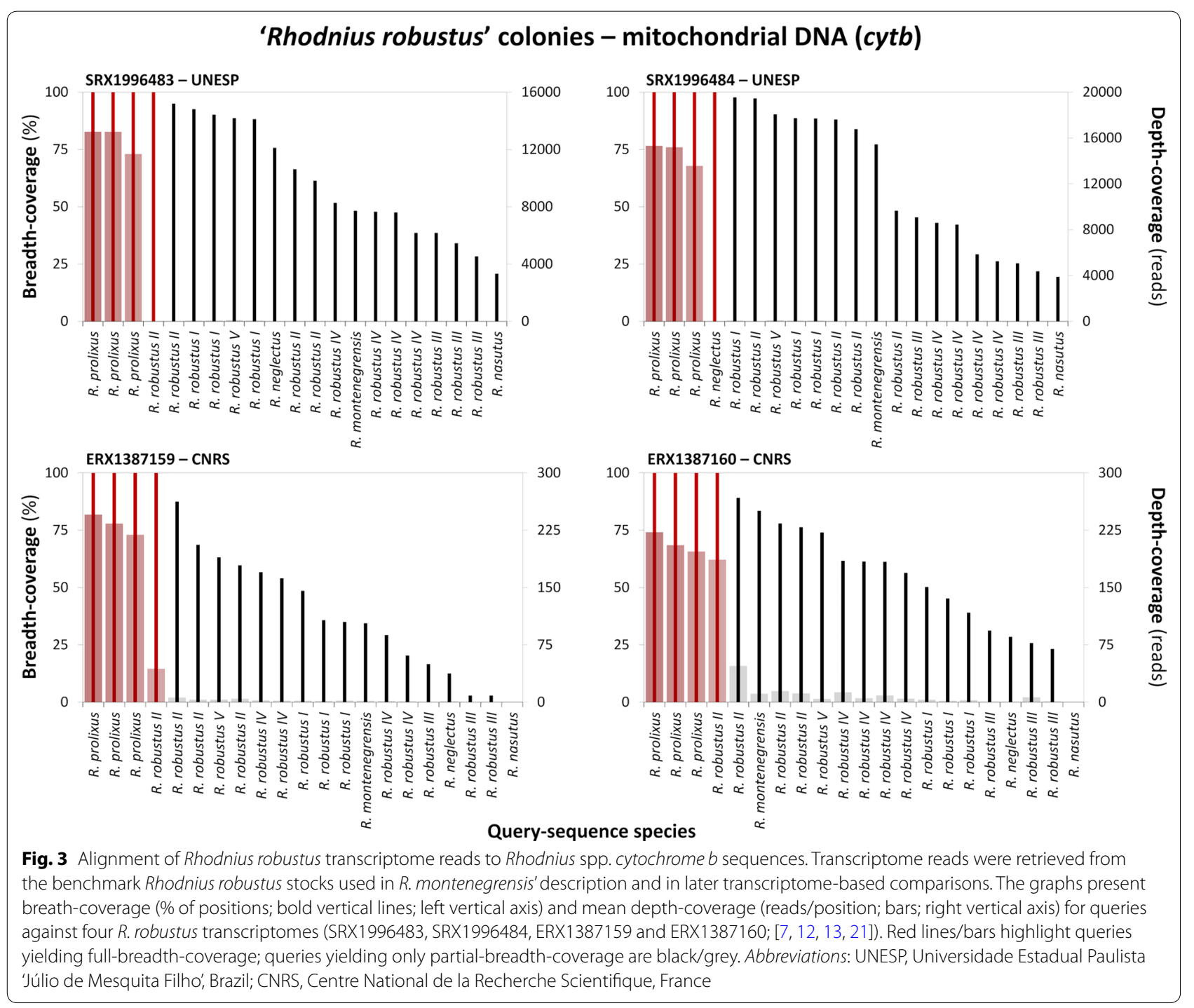

third-codon positions from the KR072682.1 sequence used to support $R$. montenegrensis as a valid species [7] (see Additional file 2: Alignment S1). Figure 3 shows that transcriptome reads from UNESP ' $R$. robustus' colonies aligned to $R$. prolixus query sequences with the highest breadth- and depth-coverage.

All the sequences in our cytb alignment, including consensus sequences generated from SRAs, comprise an open reading frame (Additional file 2: Alignment S1); this suggests that pseudogene sequences were absent from the dataset. Figure 5 shows the Bayesian $c y t b$ phylogenetic tree. This analysis recovered a well-supported clade including (i) $R$. montenegrensis' original sequence (red in Fig. 5); (ii) all previously determined $R$. robustus II sequences; and (iii) full-breadth-coverage consensus sequences generated using $R$. montenegrensis or $R$. robustus II query sequences, irrespective of whether the
SRAs were determined from UNESP colonies identified as $R$. montenegrensis or R. robustus (Fig. 5). ML and MP analyses also recovered this clade with moderate to high support (Fig. 5 and Additional file 3: Figure S1). These cytb trees also show that $R$. prolixus query sequences and the full-breadth-coverage consensus sequences they generated from ' $R$. robustus' transcriptome reads were almost identical; overall nucleotide diversity was $\pi=0.0020 \pm 0.0011 \mathrm{SE}$, with maximum divergence of just $\sim 0.3 \%$ (Table 4 ). Finally, the alignment of ' $R$. robustus' reads to the $R$. robustus II query AF421341.1 yielded two full-breadth-coverage consensus sequences that were identical to the query (Figs. 3, 5; Table 4).

From these $c y t b$ sequence-data analyses we conclude that $R$. montenegrensis is, in all likelihood, the $R$. robustus cryptic taxon dubbed ' $R$. robustus II' by Monteiro et al. [5] in 2003. In addition, we found evidence strongly 


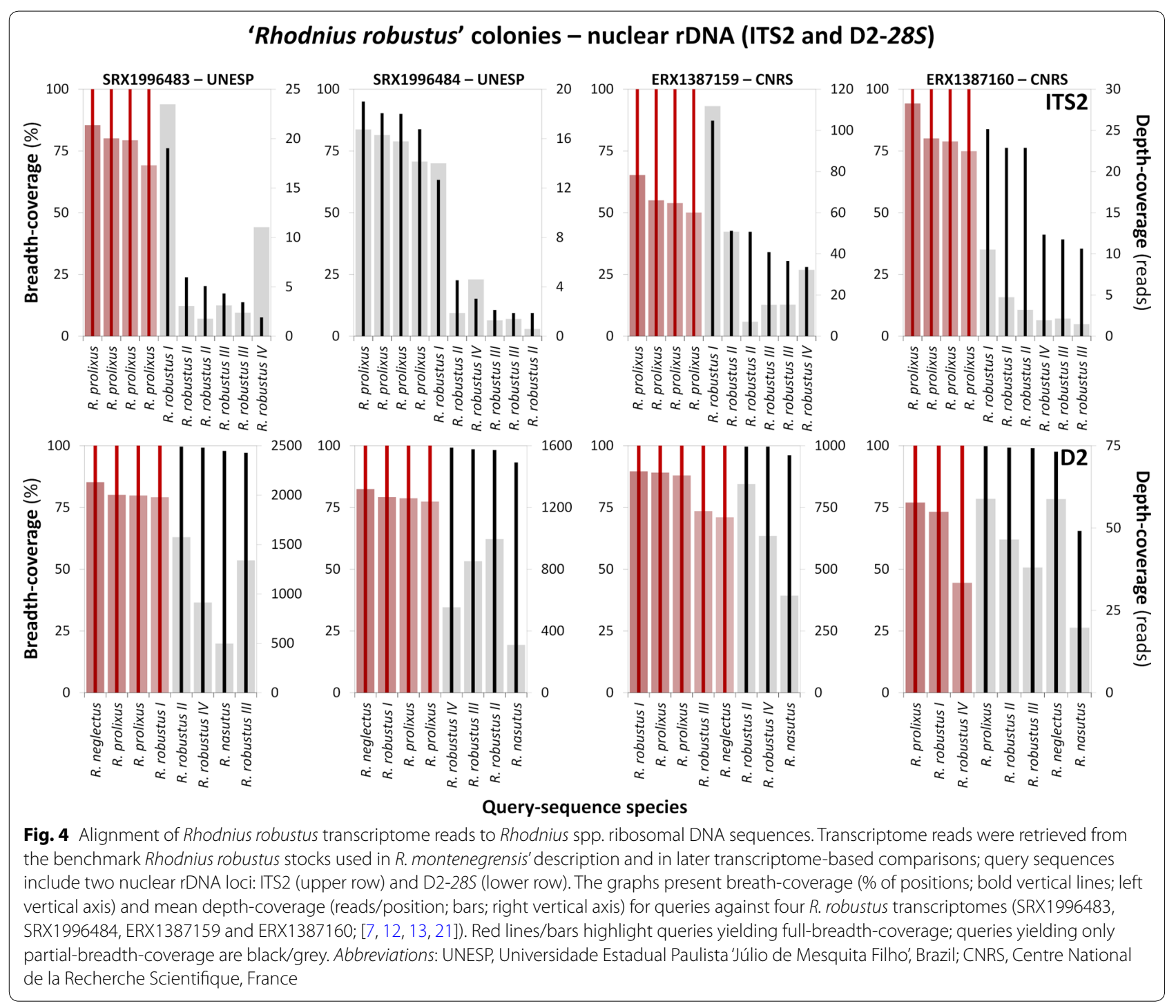

Table 2 Negligible mitochondrial cytochrome b sequence divergence between Rhodnius montenegrensis and R. robustus genotype II

\begin{tabular}{|c|c|c|c|c|c|c|c|c|c|}
\hline \multicolumn{2}{|l|}{ Sequence } & \multicolumn{3}{|l|}{ Geography } & \multicolumn{5}{|c|}{ Divergence from Rhodnius montenegrensis KR072682.1 [7] } \\
\hline GenBank ID & Reference & Locality & State/Province & Country & No. of bases & p-distance & SE & $T 3 p+\gamma$ distance & SE \\
\hline EF071583.1 & {$[30]$} & Not reported & Rondônia & Brazil & $1^{\mathrm{a}}$ & 0.00271 & 0.00258 & 0.00276 & 0.00263 \\
\hline EF011720.1 & [5] & Porto Velho & Rondônia & Brazil & 2 & 0.00542 & 0.00383 & 0.00563 & 0.00429 \\
\hline EF011724.1 & [14] & Monte Negro & Rondônia & Brazil & $3^{b}$ & 0.00813 & 0.00427 & 0.00862 & 0.00528 \\
\hline AF421341.1 & {$[5]$} & Not reported & Napo & Ecuador & 5 & 0.01355 & 0.00524 & 0.01494 & 0.00732 \\
\hline
\end{tabular}

Notes: The comparisons involve sequence KR072682.1 (from Rhodnius montenegrensis' original description [7]) vs R. robustus II sequences from the type locality (Monte Negro), from the same sub-region (state of Rondônia, Brazil), and from a 2000-km distant area (province of Napo, Ecuador) within western Amazonia

a Likely a base-call error in EF071583.1

b Including one probable base-call error in EF011724.1

Abbreviations: $\mathrm{p}$-distance, observed proportion of segregating sites; $\mathrm{SE}$, standard error (from 1000 bootstrap pseudo-replicates); T3p+ $\gamma$ distance, corrected proportion of segregating sites estimated using the best-fit model of nucleotide substitution (Tamura three-parameter model with $\gamma$-distributed rates - five categories, $\gamma=0.20$ ) 
Table 3 Full-breadth-coverage consensus sequences generated from transcriptomes determined using UNESP Rhodnius montenegrensis colonies

\begin{tabular}{|c|c|c|c|c|c|c|c|c|c|}
\hline \multirow{2}{*}{$\begin{array}{l}\text { Transcriptome } \\
\text { NCBI SRA }\end{array}$} & \multirow[t]{2}{*}{ Locus } & \multicolumn{2}{|l|}{ Query } & \multicolumn{3}{|c|}{ Depth-coverage } & \multirow{2}{*}{$\begin{array}{l}\text { Identity } \\
\text { Percent }\end{array}$} & \multicolumn{2}{|c|}{ Divergence $^{\mathrm{a}}$} \\
\hline & & Sequence & Species & Mean & Minimum & $<10$ reads $^{\mathrm{b}}(\%)$ & & Distance & SE \\
\hline \multirow[t]{9}{*}{ SRX1996481 } & \multirow[t]{5}{*}{ cytb } & EF011724.1 & Rhodnius robustus II & $13,831.35$ & 1996 & - & 99.85 & 0.00152 & 0.00144 \\
\hline & & AF421341.1 & R. robustus II & 5745.50 & 48 & - & 99.55 & 0.00463 & 0.00252 \\
\hline & & EF071583.1 & R. robustus II & 4646.05 & 67 & - & 99.70 & 0.00306 & 0.00205 \\
\hline & & EF011720.1 & R. robustus II & 4061.95 & 25 & - & 99.85 & 0.00152 & 0.00140 \\
\hline & & KR072682.1 & R. montenegrensis & 3298.10 & 12 & - & 100.00 & 0.00000 & 0.00000 \\
\hline & ITS $2^{c}$ & MK411275 & R. robustus II & 24.41 & 3 & 1.6 & 99.59 & 0.00411 & 0.00231 \\
\hline & \multirow[t]{3}{*}{ D2-28S } & AF435857.1 & R. robustus III & 631.85 & 18 & - & 100.00 & 0.00000 & 0.00000 \\
\hline & & AF435858.1 & R. robustus II & 334.82 & 2 & 1.3 & 100.00 & 0.00000 & 0.00000 \\
\hline & & AF435859.1 & R. robustus IV & 199.84 & 1 & 33.0 & 99.68 & 0.00316 & 0.00220 \\
\hline \multirow[t]{8}{*}{ SRX1996482 } & \multirow[t]{5}{*}{ cytb } & EF011724.1 & R. robustus II & $11,834.29$ & 1689 & - & 99.85 & 0.00152 & 0.00144 \\
\hline & & AF421341.1 & R. robustus II & 4834.87 & 32 & - & 99.55 & 0.00463 & 0.00252 \\
\hline & & EF071583.1 & R. robustus II & 3450.87 & 47 & - & 99.70 & 0.00306 & 0.00205 \\
\hline & & EF011720.1 & R. robustus II & 3203.49 & 13 & - & 100.00 & 0.00000 & 0.00000 \\
\hline & & KR072682.1 & R. montenegrensis & 2311.50 & 6 & 0.3 & 100.00 & 0.00000 & 0.00000 \\
\hline & ITS2 ${ }^{c}$ & MK411274 & R. robustus II & 20.29 & 1 & 29.6 & 99.32 & 0.00686 & 0.00290 \\
\hline & \multirow[t]{2}{*}{ D2-28S } & AF435857.1 & R. robustus III & 714.15 & 20 & - & 100.00 & 0.00000 & 0.00000 \\
\hline & & AF435859.1 & R. robustus IV & 211.15 & 1 & 31.3 & 99.68 & 0.00316 & 0.00205 \\
\hline
\end{tabular}

Notes: Depth-coverage (reads/position) and sequence identity (percent of identical bases) and divergence (model-based genetic distance estimates) of query vs consensus sequences are presented for three loci and two transcriptomes determined by UNESP researchers $[12,13]$

a From the best-fit models of nucleotide substitution: Tamura three-parameter with $\gamma$-distributed rates for cytb $(\gamma=0.20)$ and D2-28S $(\gamma=0.05)$, and Tamura threeparameter for ITS2; standard errors (SE) computed after 1000 bootstrap pseudo-replicates

b Percent of positions at which depth-coverage was < 10 reads (Additional file 6: Figure S4)

c See details in Additional file 1:Table S2

Abbreviations: UNESP, Universidade Estadual Paulista 'Júlio de Mesquita Filho', Brazil; NCBI, National Center for Biotechnology Information, USA; SRA, transcriptome access code at NCBI's Sequence Read Archive; cytb, mitochondrial cytochrome b gene; ITS2, second internal transcribed spacer of the nuclear ribosomal DNA; D2-28S, D2 variable region of the nuclear ribosomal DNA

suggesting that at least some of the UNESP ' $R$. robustus' colonies used as the taxonomic benchmark to infer that $R$. montenegrensis is distinct from $R$. robustus $[7,12,13]$ contain large amounts of $R$. prolixus mitochondrial DNA, and perhaps also smaller amounts of mitochondrial DNA from $R$. robustus II (i.e. R. montenegrensis) (Figs. 3, 5, Table 4).

\section{Nuclear ribosomal ITS2}

We generated 14 full-length consensus sequences from five of the six SRAs we queried with our 10 ITS2 Rhodnius spp. sequences (Tables 3, 4; Additional file 1: Tables S1, S2). Depth-coverage values were overall substantially smaller for ITS2 than for cytb transcriptome queries (Figs. 2, 3, 4 and Tables 3, 4; see also Additional file 1: Table S1 and Additional file 6: Figure S4). We generated two full-breadth-coverage consensus sequences when we aligned $R$. montenegrensis SRAs to ITS2 sequences from $R$. robustus II collected in Rondônia, Brazil (Fig. 2, Table 3$)$. We identified six variable sites $(\pi=0.005 \pm 0.002$ $\mathrm{SE}$ ) and three indels when we compared these four $R$. robustus II/R. montenegrensis sequences (Additional file 2: Alignment S2). The MK411275 (R. robustus II from Rondônia) vs SRX1996482 query generated a 99.86\% breadth-coverage (732 out of $733 \mathrm{bp}$ ) consensus sequence with mean depth-coverage of 28.06 reads (Additional file 1: Table S1). In addition, queries performed with our four $R$. prolixus ITS2 sequences generated full-breadthcoverage consensus sequences in three of the four SRAs derived from UNESP ' $R$. robustus' colonies, although depth-coverage was low for those generated from SRX1996483 (Fig. 4, Table 4, Additional file 6: Figure S4). We found just two variable sites $(\pi=0.0007 \pm 0.0006 \mathrm{SE})$ and no indels in the comparison of this subset of $R$. prolixus and ' $R$. robustus' query and consensus sequences (Additional file 2: Alignment S2).

Phylogenetic analyses recovered consistent ITS2 tree topologies (Fig. 6, Additional file 4: Figure S2). Fullbreadth-coverage consensus sequences from $R$. montenegrensis SRAs and the $R$. robustus II query sequences used to generate them clustered together in a moderately- to well-supported clade. Support was higher for the 


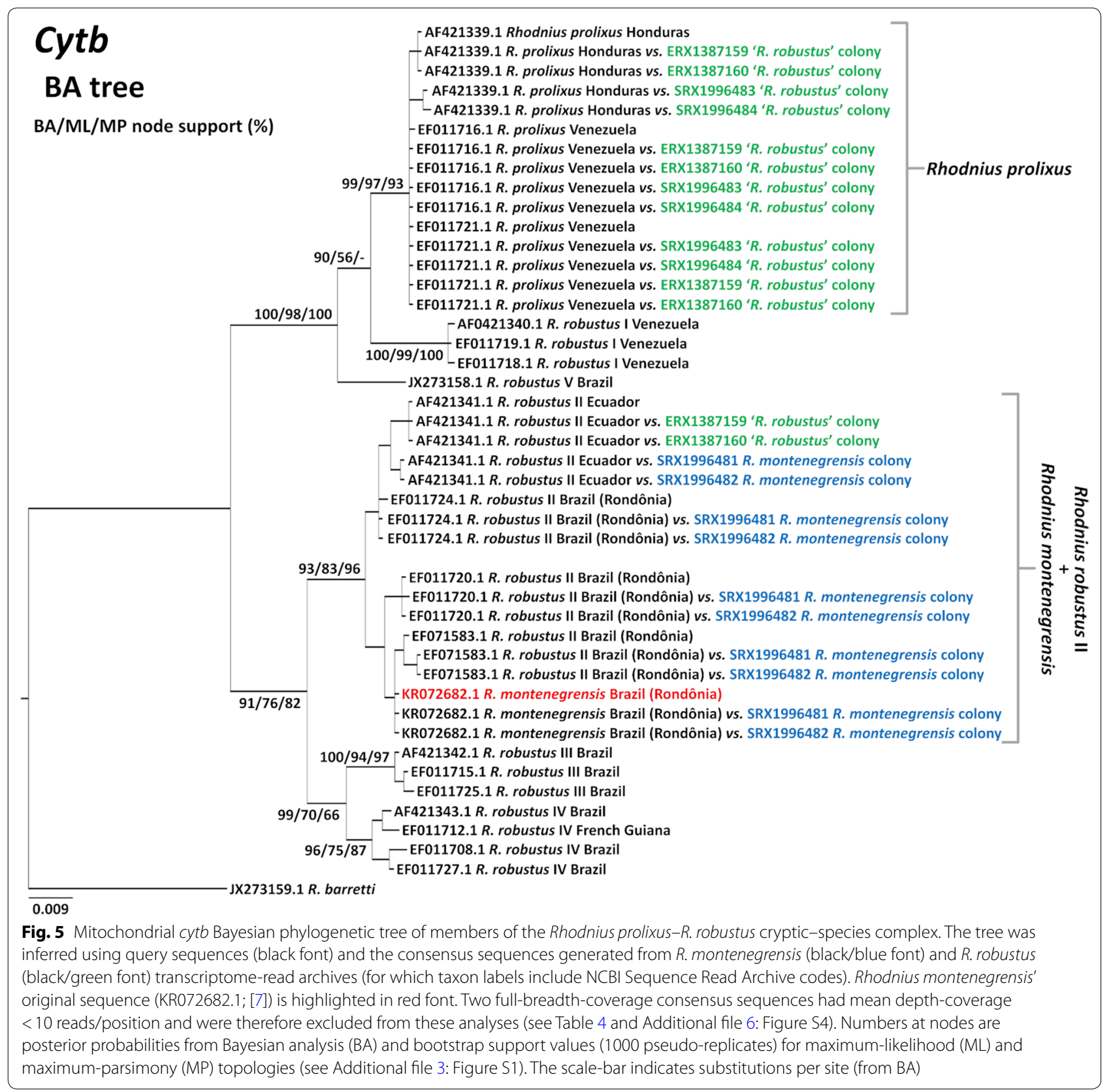

clustering of $R$. prolixus query sequences with the fullbreadth-coverage consensus sequences they generated from three ' $R$. robustus' SRAs (Fig. 6, Additional file 4: Figure S2).

We finally recall that, in the description of $R$. montenegrensis, da Rosa et al. [7] reported that BstUI cleaved ' $R$. robustus' ITS2 at one site, but did not cleave $R$. montenegrensis ITS2 amplicons; here, we found that $B s t$ UI's restriction site (CGCG) is absent from the ITS2 sequences of $R$. montenegrensis and $R$. robustus II,
III and IV, but present (sites 66-69 of our alignment; Additional file 2: Alignment S2) in those of R. robustus I and $R$. prolixus, including the latter species' genome [18], with, e.g. query sequence MK411269 ( $R$. prolixus from Guatemala; Additional file 1: Table S2) yielding a $100 \%$-identity BLASTn hit in RproC3 assembly contig ACPB03046858.1 at VectorBase (https://www.vecto rbase.org/). Thus, the BstUI digestion results in [7] suggest that bugs from UNESP ' $R$. robustus' colonies have nuclear rDNA similar to that expected in bugs of the $R$. prolixus-R. robustus I clade. 


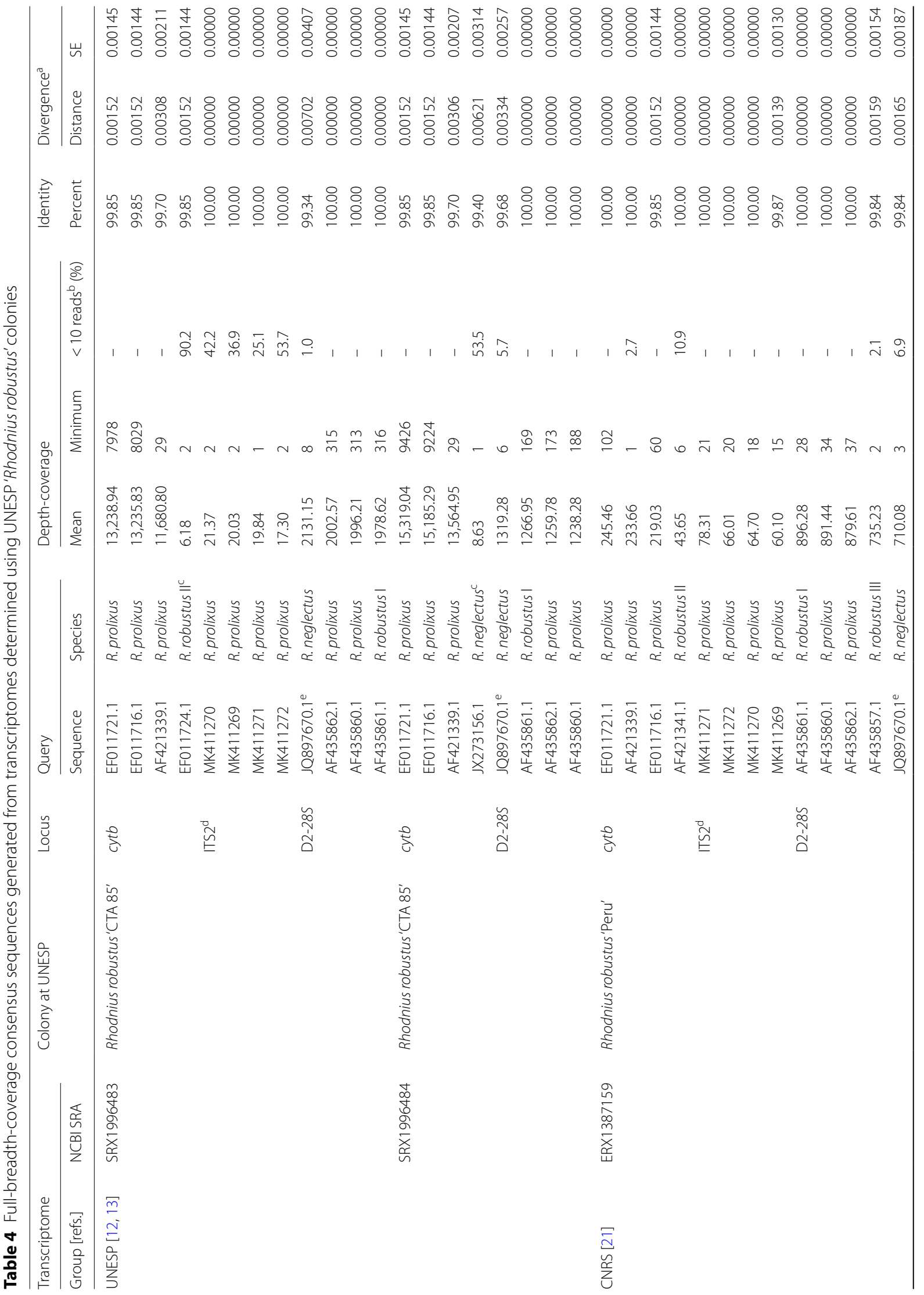




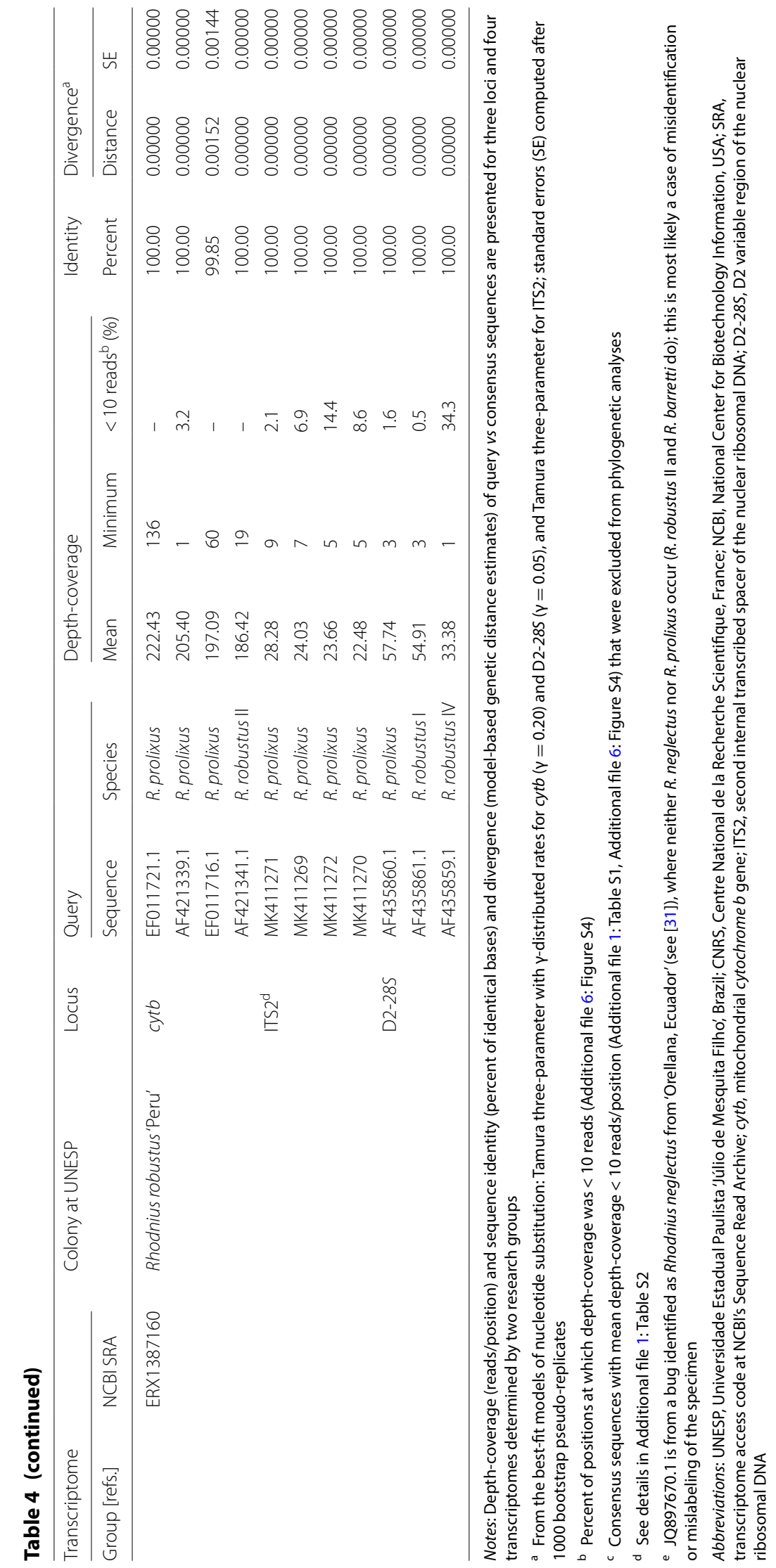




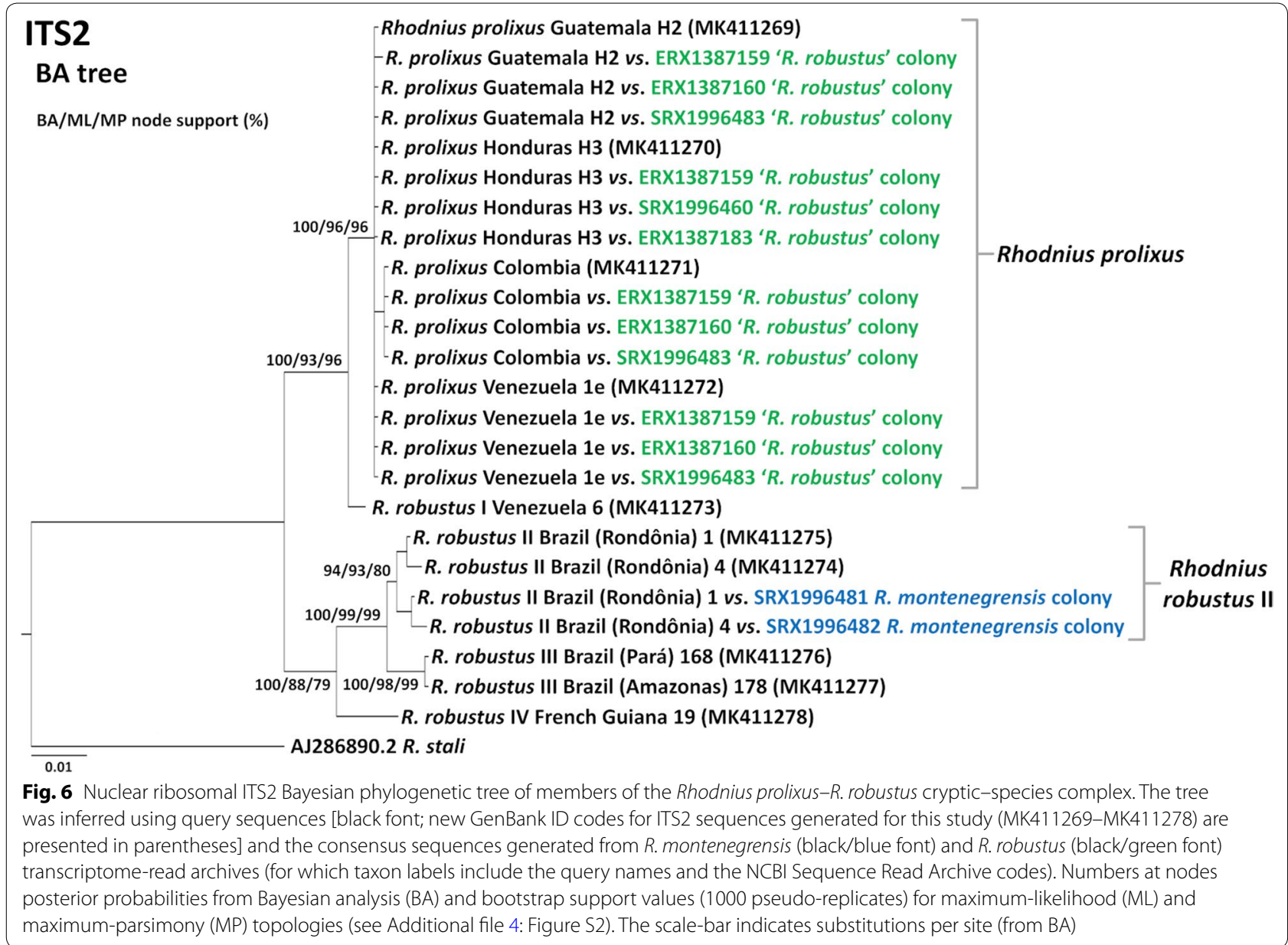

Taken together, these ITS2 data lend clear support to the $c y t b$-based findings described above. In particular, they (i) confirm that $R$. montenegrensis is genetically indistinguishable from $R$. robustus II, and (ii) further suggest that the UNESP ' $R$. robustus' colonies used as the taxonomic benchmark to conclude that $R$. montenegrensis is distinct from $R$. robustus [7, 12, 13] are mainly or fully composed of bugs with $R$. prolixus DNA, with no detectable $R$. robustus nuclear ITS2 sequences.

\section{Nuclear ribosomal D2-28S}

Our D2-28S $R$. prolixus-R. robustus complex query sequences generated 21 full-breadth-coverage sequences from the six transcriptome SRAs; depth-coverage was overall substantial (typically $>250$ reads/position on average) except for queries against SRA ERX1387160 (Figs. 2, 4; Tables 3, 4). Depth-coverage was, however, low over certain stretches of some full-breadth-coverage consensus sequences (Tables 3, 4; Additional file 6: Figure S4). When we used $R$. robustus IV's AF435859.1 as the query, for example, mean depth-coverage was high for the consensus sequences generated from $R$. montenegrensis' SRAs, yet depth-coverage fell to $<10$ reads/ position between positions $\sim 260$ and $\sim 470$ (Table 3, Additional file 6: Figure S4). Similarly, the AF435859.1 vs ERX1387160 query generated a full-breadth-coverage consensus sequence with 217 positions (34.3\%) supported by $<10$ reads each (Table 4, Additional file 6: Figure S4). We therefore regard the consensus sequences generated using our $R$. robustus IV query as dubious. Queries using the $R$. robustus III sequence generated fullbreadth-coverage sequences from the two $R$. montenegrensis SRAs (with substantial depth-coverage; Fig. 2) and from one ' $R$. robustus' SRA (Fig. 4), for which depth-coverage was $<100$ reads/position at 103 positions, including a stretch with $<10$ reads/position between positions 539 and 551 (Table 4, Additional file 6: Figure S4). We note that position 540 in our 633-bp alignment (Additional file 2: Alignment S3) is the variable position that separates the clade including $R$. robustus II-IV (all with $\mathrm{T}$ ) and that including $R$. prolixus and $R$. robustus I (with a derived C) [5]. We generated one full-length sequence 
using $R$. robustus II from Ecuador (AF435858.1) as the query (Fig. 2); depth-coverage was high except for a 70-position section with depth-coverage below 100 reads/position, including $<20$ reads at positions 351-369 and $<10$ reads at nine positions within this 19-bp stretch, which includes three variable positions (Table 3, Additional file 2: Alignment S3 and Additional file 6: Figure S4).

Our R. prolixus (AF435860.1 and AF435862.1) and $R$. robustus I (AF435861.1) D2-28S query sequences were identical and generated full-breadth-coverage consensus sequences from all ' $R$. robustus' SRAs (Fig. 4); the exception was $R$. prolixus query AF435862.1 vs ERX1387160, for which no read aligned to the last base (Table 4, Additional file 1: Table S1). These full-breadth-coverage consensus sequences did not differ by a single base from the also identical query sequences. Depth-coverage was overall high (except, as mentioned above, for SRA ERX1387160), with only a few, short stretches having low depth-coverage (Table 4, Additional file 6: Figure S4).

D2-28S sequences produced gene trees with relatively poor resolution; although the clustering of query and consensus sequences overall mirrored the patterns described for $c y t b$ and ITS2 (Figs. 5, 6), most tree nodes had low to very low support (Additional file 5: Figure S3). Overall, these D2-28S data further suggest that the benchmark UNESP's ' $R$. robustus' colonies are mainly or fully composed of bugs with $R$. prolixus DNA, with only one $R$. robustus query (genotype IV) generating a fullbreadth-coverage, yet low-depth-coverage, consensus sequence. The data were less informative with regard to the relation between $R$. montenegrensis and $R$. robustus II, likely because the only $R$. robustus II D2-28S sequence so far available (AF435858.1) is from an Ecuadorian bug caught $\sim 2000 \mathrm{~km}$ from $R$. montenegrensis' type locality. Our finding that reads from three ' $R$. robustus' SRAs aligned with full-breadth- and high depth-coverage to one $R$. neglectus query (Fig. 4, Table 4) is discussed in the next sub-section.

\section{Quality check: cytb, D2-28S and AmpG}

As expected, no full-breadth-coverage sequences were generated when we aligned our study SRAs to R. nasutus sequences or to $A m p G$ sequences from members of the $R$. prolixus $-R$. robustus species complex (Additional file 1: Table S1). Rhodnius neglectus queries, however, generated four full-breadth-coverage consensus sequences (Figs. 3, 4, Table 4). One R. neglectus cytb query (JX273156.1) yielded full-breadth-coverage against one ' $R$. robustus' SRA, yet with mean depth-coverage $<10$ reads/position (Fig. 3, Table 4). In contrast, the only available $R$. neglectus D2-28S query [31] yielded both fullbreadth-coverage and substantial mean depth-coverage (albeit with a $\sim 45$-bp stretch with depth-coverage $<20$ reads/position) against three ' $R$. robustus' SRAs (Fig. 4, Table 4). This D2-28S sequence (JQ897670.1) is from a bug identified as $R$. neglectus but reportedly collected in western Amazonia ('Orellana, Ecuador') [31], where $R$. neglectus does not occur $[9,10]$. Therefore, this is most likely a case of misidentification or mislabeling of the specimen (voucher 'UCR_ENT_00052203' at the Entomology Collection of the University of California, Riverside) [31] (see Table 4).

\section{Discussion}

In this report we have illustrated how publicly available transcriptome data can be used to clarify the systematics of a taxonomically challenging group of cryptic diseasevector species. This transcriptome-based approach to molecular systematics has, to our knowledge, not been used before in vector studies; it is overall analogous to the assembly of mitochondrial genes from transcriptome data used to study, for example, poison frogs [32], catfish [33], true bugs [34] or ants [35] (see also [36]). We found evidence confirming that $R$. montenegrensis, a species described in 2012 [7], is genetically indistinguishable from $R$. robustus II, one of the sibling taxa within $R$. robustus (s.l.) that Monteiro and colleagues discovered in $2003[5,6]$. To solve the paradox that $R$. montenegrensis appears to be morphologically and genetically distinct from $R$. robustus $[7,12,13]$, we then showed that the ' $R$. robustus' stocks used as the taxonomic benchmark in $R$. montenegrensis' description [7] and in later transcriptome-based comparisons $[12,13]$ are almost certainly $R$. prolixus, likely mixed to some degree with $R$. robustus.

We note that our confirmation that $R$. montenegrensis and $R$. robustus II are almost identical genetically does not invalidate the former as a separate species - it just shows that 'Rhodnius montenegrensis' is the binomial for what we informally called 'Rhodnius robustus II' [5, 6]. Our results, in any case, provide an example of how triatomine-bug taxonomic research can be confounded when sequence-data analyses are loosely interpreted [6]. Thus, the cytb data presented in the description of $R$. montenegrensis [7] already showed that $R$. montenegrensis and $R$. robustus II are all but indistinguishable at that locus (Table 2). However, instead of pointing out the virtual identity of $R$. montenegrensis and $R$. robustus II sequences, da Rosa et al. [7] underscored the (effectively negligible) differences - a stance later mirrored in a study involving $c y t b$-based identification of $R$. montenegrensis specimens [37]. As noted by Monteiro et al. [6], this is probably also the case for $R$. marabaensis and $R$. robustus III from southeastern Amazonia [5]; $R$. marabaensis sequences, however, have not been made available in public databases [8]. Along the same lines, the 
hypothesis that $R$. milesi [38] and $R$. taquarussuensis [39] are $R$. neglectus variants (see $[6,10]$ ) recently received empirical support from molecular systematics $[6,40]$. A further example of taxonomic uncertainty is $R$. zeledoni [41], whose holotype (the only known specimen) is strikingly similar to the sympatric $R$. domesticus $[1,6,42]$; however, the data needed to address this uncertainty are so far unavailable.

Our results also show how the use of mixed or misidentified Rhodnius spp. colonies can confound taxonomic research even further - and how we can take advantage of public-domain molecular data to clarify cryptic-species systematics in the face of such confusion. Perhaps more importantly, our finding that some putative ' $R$. robustus' colonies are fully or almost fully composed of bugs with $R$. prolixus DNA contributes to casting doubts over the conclusions of the many studies that made use of non-genotyped ' $R$. prolixus' or ' $R$. robustus' laboratory stocks. Mesquita et al. [18] noted this problem in their quest for a pure $R$. prolixus stock to be used in genome sequencing. Of the 15 putative $R$. prolixus colonies they genotyped, just four were pure: one had both mitochondrial and nuclear $R$. robustus II DNA and ten had introgressed $R$. robustus IV mitochondrial DNA (see p. 28 of Appendix SI of [18]). As transcriptome (and genome) data from other putative $R$. prolixus and $R$. robustus colonies progressively accrue, approaches analogous to the one we illustrate here may help elucidate their taxonomic identity and genetic integrity. This would be particularly interesting in the case of putative $R$. prolixus colonies derived from the legendary stock used by Sir Vincent B Wigglesworth in his seminal studies on insect physiology [43], but would also be valuable for assessing the taxonomic status of bugs used in more recent research on Rhodnius spp. morphology, genetics, physiology, behavior, bionomics or interactions with microorganisms including Trypanosoma cruzi (e.g. [44-48]). Similarly, our results suggest that the value of several approaches put forward to investigate the systematics of cryptic or near-cryptic Rhodnius taxa (including, for example, the use of quantitative phenotypic traits $[7,49]$ or ctyogenetics $[39,50])$ will have to be reappraised after careful consideration of the specific status of the bugs, whether field-collected or laboratory-reared, used in comparative analyses.

\section{Conclusions}

Here, we have illustrated how public-domain transcriptome reads and locus-specific sequences can be combined to address challenging issues in vector systematics. Using query sequences from mitochondrial and nuclear loci, six publicly-available raw transcriptome datasets, and a straightforward bioinformatics approach, we (i) confirmed that $R$. montenegrensis and $R$. robustus II are in all likelihood the same species, and (ii) showed that the UNESP ' $R$. robustus' stocks used as the taxonomic benchmark in $R$. montenegrensis' description and in later comparative studies are most likely a mixture of (mainly) $R$. prolixus and (partly) $R$. robustus (probably genotype II). In this particular instance of taxonomic confusion, misinterpretation of sequence-data analyses was compounded by the misidentification of taxonomic-benchmark laboratory stocks. More generally, and together with previous reports of mixed and/or misidentified Rhodnius spp. colonies, our results call into question the conclusions of many studies based on non-genotyped ' $R$. prolixus' or ' $R$. robustus' stocks. Rhodnius prolixus and $R$. robustus (s.l.) are similar in many respects, but differ in a fundamental way: the former is a primary domestic vector of Chagas disease, whereas the latter comprises a suite of sylvatic species (including $R$. montenegrensis) of limited medical relevance. Correct species identification will be key to any attempt at understanding what physiological, behavioral or ecological factors may underlie this crucial difference.

\section{Additional files}

Additional file 1: Table S1. Details on transcriptome-read queries. Table S2. ITS2 sequences generated in this study.

Additional file 2: Alignment S1. Cytb sequences. Alignment S2. ITS2 sequences. Alignment S3. D2-28S sequences.

Additional file 3: Figure S1. Cytb trees of members of the Rhodnius prolixus-R. robustus complex. Maximum-likelihood (ML; Tamura 3-parameter $+\gamma$ ) and maximum-parsimony (MP) trees using query sequences (black) and consensus sequences from $R$. montenegrensis (black/blue) and R. robustus (black/green) transcriptome-read archives (with NCBI codes). Rhodnius montenegrensis' original sequence (KR072682.1; [7]) highlighted in red. Consensus sequences with mean depth-coverage $<10$ reads/position were excluded (Table 4, Additional file 6: Figure S4). Node-support: 1000 bootstrap pseudo-replicates. Scalebars: substitutions/site (ML) and number of substitutions (MP).

Additional file 4: Figure S2. ITS2 trees of members of the Rhodnius prolixus-R. robustus complex. Maximum-likelihood (ML; Tamura three-parameter) and maximum-parsimony (MP) inferred using query sequences (black) and consensus sequences from R. montenegrensis (black/blue) and $R$. robustus (black/green) transcriptome-read archives (with NCBI codes). Node-support: 1000 bootstrap pseudo-replicates. Scale-bars: substitutions/site (ML) and number of substitutions (MP).

Additional file 5: Figure S3. D2-28S phylogenetic trees of members of the Rhodnius prolixus-R. robustus complex and related taxa. Bayesian analysis (BA), maximum-likelihood ( $M L$; Tamura 3-parameter $+\gamma$ ) and maximum parsimony (MP) trees based on query sequences (black) and consensus sequences from $R$. montenegrensis and $R$. robustus transcriptome-read archives (with NCBI codes). JQ897670.1 is from a bug identified as 'Rhodnius neglectus' from 'Orellana, Ecuador' [31], where R. neglectus does not occur; most likely misidentification/mislabeling. Node-support: posterior probabilities (BA) and 1000 bootstrap pseudo-replicates (ML/MP). Scalebars: substitutions/site (BA and $M L$ ) and number of substitutions (MP). 
Additional file 6: Figure S4. Depth-coverage for full-breadth-coverage consensus sequences in which $\geq 1$ position had depth-coverage $<10$ reads. Red: sequences with mean depth-coverage $<10$ reads/position (regarded as unreliable and excluded from phylogenetic analyses); orange: sequences with mean depth-coverage $\geq 10$ reads/position, but with $\geq 15 \%$ of positions supported by $<10$ reads (regarded as dubious); green: sequences with only short stretches ( $<15 \%$ of sequence length) with depth-coverage was $<10$ reads (reliable). Y-axes on a $\log 10$ scale.

\section{Abbreviations}

AmpG: amplicon G (a putative nuclear intron); BA: Bayesian (phylogenetic) analyses; BIC: Bayesian information criterion; CNRS: Centre National de la Recherche Scientifique; cytb: mitochondrial cytochrome b gene; D2-28S: D2 variable region of the 285 of the nuclear rDNA; EMBL-EBI: European Molecular Biology Laboratory, European Bioinformatics Institute; ENA: European Nucleotide Archive; ITS2: second internal transcribed spacer of the nuclear rDNA; ML: maximum likelihood; MP: maximum parsimony; NCBI: National Center for Biotechnology Information; rDNA: ribosomal DNA; SRA: Sequence Read Archive; T3p: Tamura three-parameter model of nucleotide substitution; UNESP: Universidade Estadual Paulista 'úlio de Mesquita Filho'.

\section{Acknowledgements}

We thank the Bioinformatics Platform at the Instituto René Rachou, Fiocruz Minas Gerais, for providing the bioinformatics computational resources for this project.

\section{Authors' contributions}

FA-F conceived the study with input from FAM and CL. RNB, JAG and RCMS queried transcriptome SRAs, generated consensus sequences and computed coverage. FAM and CL contributed ITS2 query sequences. FA-F, RNB, JAG and $\mathrm{CL}$ analyzed the data. FA-F and RNB drafted the first version of the manuscript. All authors contributed to the interpretation of results, read, commented on the manuscript drafts. All authors read and approved the final manuscript.

\section{Funding}

The present study was funded by the following: Programa de Pós-Graduação em Ciências da Saúde, Instituto René Rachou (Fiocruz); Fundação de Amparo à Pesquisa do Estado de Minas Gerais (FAPEMIG); Coordenação de Aperfeiçoamento de Pessoal de Nível Superior (CAPES), Brazilian Ministry of Education; and Vice-Presidência de Pesquisa e Coleções Biológicas, Fiocruz. The funders had no role in the design of the study, in the collection, analysis or interpretation of the data, or in writing the manuscript.

\section{Availability of data and materials}

Data supporting the conclusions of this article are included in the article and its additional files. The newly generated sequences were submitted to the GenBank database under the accession numbers MK411269-MK411278.

\section{Ethics approval and consent to participate}

Not applicable.

\section{Consent for publication}

Not applicable.

\section{Competing interests}

The authors declare that they have no competing interests.

\section{Author details}

${ }^{1}$ Grupo Triatomíneos, Instituto René Rachou, Fiocruz Minas Gerais, Fundação Oswaldo Cruz (Fiocruz), Belo Horizonte, Brazil. ${ }^{2}$ Programa Interunidades de Pós-Graduação em Bioinformática, Universidade Federal de Minas Gerais, Belo Horizonte, Brazil. ${ }^{3}$ Instituto René Rachou, Fiocruz Minas Gerais, Fundação Oswaldo Cruz (Fiocruz), Belo Horizonte, Brazil. ${ }^{4}$ Laboratório de Epidemiologia e Sistemática Molecular, Instituto Oswaldo Cruz, Fundação Oswaldo Cruz (Fiocruz), Rio de Janeiro, Brazil. ${ }^{5}$ Instituto de Biologia, Universidade Federal do Rio de Janeiro, Rio de Janeiro, Brazil. ${ }^{6}$ Programa de Pós-Graduação em Medicina Tropical, Núcleo de Medicina Tropical, Faculdade de Medicina, Universidade de Brasília, Brasilia, Brazil.
Received: 22 January 2019 Accepted: 9 June 2019

Published online: 17 June 2019

\section{References}

1. Lent H, Wygodzinsky P. Revision of the Triatominae (Hemiptera, Reduviidae) and their significance as vectors of Chagas' disease. Bull Am Mus Nat Hist. 1979;163:125-520.

2. Barrett TV. Advances in triatomine bug ecology in relation to Chagas disease. In: Harris KH, editor. Advances in disease vector research, vol. 8. New York: Springer; 1991. p. 143-76.

3. Harry M. Isozymic data question the specific status of some blood-sucking bugs of the genus Rhodnius, vectors of Chagas disease. Trans R Soc Trop Med Hyg. 1993;87:492-3.

4. Monteiro FA, Escalante AA, Beard CB. Molecular tools and triatomine systematics: a public health perspective. Trends Parasitol. 2001;17:344-7.

5. Monteiro FA, Barrett TV, Fitzpatrick S, Cordon-Rosales C, Feliciangeli D, Beard CB. Molecular phylogeography of the Amazonian Chagas disease vectors Rhodnius prolixus and R. robustus. Mol Ecol. 2003;12:997-1006.

6. Monteiro FA, Weirauch C, Felix M, Lazoski C, Abad-Franch F. Evolution, systematics, and biogeography of the Triatominae, vectors of Chagas disease. Adv Parasitol. 2018;99:265-344.

7. da Rosa JA, Rocha CS, Gardim S, Pinto MC, Mendonça VJ, Ferreira Filho JCR, et al. Description of Rhodnius montenegrensis sp. nov. (Hemiptera: Reduviidae: Triatominae) from the state of Rondônia, Brazil. Zootaxa. 2012;3478:62-76

8. Souza ED, Von Atzingen NC, Furtado MB, de Oliveira J, Nascimento JD, Vendrami DP, et al. Description of Rhodnius marabaensis sp. n. (Hemiptera, Reduviidae, Triatominae) from Pará State, Brazil. Zookeys. 2016;621:45-62.

9. Abad-Franch F, Monteiro FA. Biogeography and evolution of Amazonian triatomines (Heteroptera: Reduviidae): implications for Chagas disease surveillance in humid forest eco-regions. Mem Inst Oswaldo Cruz. 2007;102:57-69.

10. Abad-Franch F, Monteiro FA, Jaramillo ON, Gurgel-Gonçalves R, Dias FBS, Diotaiuti L. Ecology, evolution, and the long-term surveillance of vectorborne Chagas disease: a multi-scale appraisal of the tribe Rhodniini (Triatominae). Acta Trop. 2009;110:159-77.

11. Fitzpatrick S, Feliciangeli MD, Sánchez-Martín MJ, Monteiro FA, Miles MA. Molecular genetics reveal that silvatic Rhodnius prolixus do colonise rural houses. PLoS Negl Trop Dis. 2008;2:e210.

12. de Carvalho DB, Congrains C, Chahad-Ehlers S, Pinotti H, Brito RA, da Rosa JA. Differential transcriptome analysis supports Rhodnius montenegrensis and Rhodnius robustus (Hemiptera, Reduviidae, Triatominae) as distinct species. PLoS ONE. 2017;12:e0174997.

13. de Carvalho DB. Estudo de transcriptomas por RNAseq em tecidos de cabeça e glândula salivar de Rhodnius montenegrensis e Rhodnius robustus (Hemiptera, Reduviidade, Triatominae). PhD Thesis, Universidade Estadual Paulista'Júlio de Mesquita Filho'; 2016. https://repositorio.unesp.br/handl e/11449/137929. Accessed 07 Jan 2019.

14. Pavan MG, Monteiro FA. A multiplex PCR assay that separates Rhodnius prolixus from members of the Rhodnius robustus cryptic species complex (Hemiptera: Reduviidae). Trop Med Int Health. 2007;12:751-8.

15. Pavan MG, Mesquita RD, Lawrence GG, Lazoski C, Dotson EM, Abubucker $\mathrm{S}$, et al. A nuclear single-nucleotide polymorphism (SNP) potentially useful for the separation of Rhodnius prolixus from members of the Rhodnius robustus cryptic species complex (Hemiptera: Reduviidae). Infect Genet Evol. 2013;14:426-33.

16. Abad-Franch F, Pavan MG, Jaramillo-O N, Palomeque FS, Dale C, Chaverra D, et al. Rhodnius barretti, a new species of Triatominae (Hemiptera: Reduviidae) from western Amazonia. Mem Inst Oswaldo Cruz. 2013;108(Suppl. 1):92-9.

17. Barrett TV. Species interfertility and crossing experiments in triatomine systematics. In: Schofield CJ, Dujardin J-P, Jurberg J, editors. Proceedings of the international workshop on population genetics and control of Triatominae, Santo Domingo de los Colorados, Ecuador, Sept. 1995. Mexico City: INDRE; 1996. p. 72-7.

18. Mesquita RD, Vionette-Amaral RJ, Lowenberger C, Rivera-Pomar R, Monteiro FA, Minx P, et al. Genome of Rhodnius prolixus, an insect vector 
of Chagas disease, reveals unique adaptations to hematophagy and parasite infection. Proc Natl Acad Sci USA. 2015;1 12:14936-41.

19. Monteiro FA, Wesson DM, Dotson EM, Schofield CJ, Beard CB. Phylogeny and molecular taxonomy of the Rhodniini derived from mitochondrial and nuclear DNA sequences. Am J Trop Med Hyg. 2000;62:460-5.

20. Marcilla A, Bargues MD, Ramsey JM, Magallon-Gastelum E, SalazarSchettino PM, Abad-Franch F, et al. The ITS2 of the nuclear rDNA as a molecular marker for populations, species, and phylogenetic relationships in Triatominae (Hemiptera: Reduviidae), vectors of Chagas disease. Mol Phylogenet Evol. 2001;18:136-42.

21. Marchant A. Le processus de domiciliation des punaises hématophages vectrices de la maladie de Chagas: apport de l'étude du transcriptome chimiosensoriel. PhD Thesis. Paris: Université Paris-Saclay; 2016. https://tel. archives-ouvertes.fr/tel-01350868/. Accessed 7 Jan 2019.

22. Li H, Durbin R. Fast and accurate short read alignment with BurrowsWheeler transform. Bioinformatics. 2009;25:1754-60.

23. Li H, Handsaker B, Wysoker A, Fennell T, Ruan J, Homer N, et al. The sequence alignment/map format and SAMtools. Bioinformatics. 2009;25:2078-9

24. Okonechnikov K, Golosova O, Fursov M, UGENE team. Unipro UGENE: a unified bioinformatics toolkit. Bioinformatics. 2012;28:1166-7.

25. Quinlan AR, Hall IM. BEDTools: a flexible suite of utilities for comparing genomic features. Bioinformatics. 2010;26:841-2.

26. Katoh K, Standley DM. MAFFT multiple sequence alignment software version 7: improvements in performance and usability. Mol Biol Evol. 2013;30:772-80

27. Kumar S, Stecher G, Li M, Knyaz C, Tamura K. MEGA X: molecular evolutionary Genetics Analysis across computing platforms. Mol Biol Evol. 2018:35:1547-9.

28. Suchard MA, Lemey P, Baele G, Ayres DL, Drummond AJ, Rambaut A. Bayesian phylogenetic and phylodynamic data integration using BEAST 1.10. Virus Evol. 2018;4:vey016.

29. Todd EV, Black MA, Gemmell NJ. The power and promise of RNA-seq in ecology and evolution. Mol Ecol. 2016;25:1224-41.

30. Maia da Silva F, Junqueira AC, Campaner M, Rodrigues AC, Crisante G, Ramírez LE, et al. Comparative phylogeography of Trypanosoma rangeli and Rhodnius (Hemiptera: Reduviidae) supports a long coexistence of parasite lineages and their sympatric vectors. Mol Ecol. 2007;16:3361-73.

31. Hwang WS, Weirauch C. Evolutionary history of assassin bugs (Insecta: Hemiptera: Reduviidae): insights from divergence dating and ancestral state reconstruction. PLoS ONE. 2012;7:e45523.

32. Lyra ML, Sanchez E, Künzel S, Lötters S, Haddad CFB, Vences M. The mitochondrial genomes of three species of poison frogs (Anura: Dendrobates). Mitochondr DNA B Resour. 2017;2:397-9.

33. Moreira DA, Furtado C, Parente TE. The use of transcriptomic next-generation sequencing data to assemble mitochondrial genomes of Ancistrus spp. (Loricariidae). Gene. 2015;573:171-5.

34. Song N, Zhang H, Li H, Cai W. All 37 Mitochondrial genes of aphid Aphis craccivora obtained from transcriptome sequencing: implications for the evolution of aphids. PLoS ONE. 2016;11:e0157857.

35. Vieira GA, Prosdocimi F. Accessible molecular phylogenomics at no cost: obtaining 14 new mitogenomes for the ant subfamily Pseudomyrmecinae from public data. PeerJ. 2019;7:e6271.

36. Plese B, Rossi ME, Kenny N, Taboada S, Koutsouveli V, Riesgo A. Trimitomics: an efficient pipeline for mitochondrial assembly from transcriptomic reads in non-model species. bioRxiv. 2018. https://doi.org/10.1101/413138.

37. Bilheiro AB, da Rosa JA, de Oliveira J, Belintani T, Fontes G, Medeiros JF, et al. First report of natural infection with Trypanosoma cruzi in Rhodnius montenegrensis (Hemiptera, Reduviidae, Triatominae) in western Amazon, Brazil. Vector Borne Zoonotic Dis. 2018;18:605-10.

38. Valente VC, Valente SAS, Carcavallo RU, Rocha DS, Galvão C, Jurberg J. Considerações sobre uma nova espécie do gênero Rhodnius Stål, do Estado de Pará, Brasil (Hemiptera: Reduviidae: Triatominae). Entomol Vectores. 2001;8:65-80.

39. da Rosa JA, Justino HHG, Nascimento JD, Mendonça VJ, Rocha CS, de Carvalho DB, et al. A new species of Rhodnius from Brazil (Hemiptera, Reduviidae, Triatominae). Zookeys. 2017:675:1-25.

40. Nascimento JD, da Rosa JA, Salgado-Roa FC, Hernández C, Pardo-Diaz C, Alevi KCC, et al. Taxonomical over splitting in the Rhodnius prolixus (Insecta: Hemiptera: Reduviidae) clade: are R. taquarussuensis (da Rosa et al., 2017) and R. neglectus (Lent, 1954) the same species? PLoS ONE. 2019;14:e0211285.

41. Jurberg J, Rocha DS, Galvão C. Rhodnius zeledoni sp. nov. afim de Rhodnius paraensis Sherlock, Guitton \& Miles, 1977 (Hemiptera, Reduviidae, Triatominae). Biota Neotrop. 1977;2009(9):123-8.

42. Jurberg J, Rodrigues JMS, Moreira FFF, Dale C, Cordeiro IRS, Lamas JRVD, et al. Atlas iconográfico dos triatomíneos do Brasil—vetores da doença de Chagas. Rio de Janeiro: Instituto Oswaldo Cruz - Fiocruz; 2014.

43. Wigglesworth VB. The principles of insect physiology. London: Chapman and Hall; 1972

44. da Rosa JA, Mendonça VJ, Gardim S, de Carvalho DB, de Oliveira J, Nascimento JD, et al. Study of the external female genitalia of 14 Rhodnius species (Hemiptera, Reduviidae, Triatominae) using scanning electron microscopy. Parasite Vectors. 2014;7:17.

45. Paluzzi JP, Park Y, Nachman RJ, Orchard I. Isolation, expression analysis, and functional characterization of the first antidiuretic hormone receptor in insects. Proc Natl Acad Sci USA. 2010;107:10290-5.

46. Lahondère C, Insausti TC, Paim RM, Luan X, Belev G, Pereira MH, et al. Countercurrent heat exchange and thermoregulation during bloodfeeding in kissing bugs. Elife. 2017;6:e26107.

47. de la Vega GJ, Schilman PE. Using eco-physiological traits to understand the realized niche: the role of desiccation tolerance in Chagas disease vectors. Oecologia. 2017;185:607-18.

48. Takano-Lee M, Edman JD. Lack of manipulation of Rhodnius prolixus (Hemiptera: Reduviidae) vector competence by Trypanosoma cruzi. J Med Entomol. 2002;39:44-51.

49. Gurgel-Gonçalves R, Abad-Franch F, Ferreira JB, Santana DB, Cuba CA. Is Rhodnius prolixus (Triatominae) invading houses in central Brazil? Acta Trop. 2008;107:90-8.

50. Pita S, Panzera F, Ferrandis I, Galvão C, Gómez-Palacio A, Panzera Y. Chromosomal divergence and evolutionary inferences in Rhodniini based on the chromosomal location of ribosomal genes. Mem Inst Oswaldo Cruz. 2013;108:376-82.

\section{Publisher's Note}

Springer Nature remains neutral with regard to jurisdictional claims in published maps and institutional affiliations.

Ready to submit your research? Choose BMC and benefit from:

- fast, convenient online submission

- thorough peer review by experienced researchers in your field

- rapid publication on acceptance

- support for research data, including large and complex data types

- gold Open Access which fosters wider collaboration and increased citations

- maximum visibility for your research: over 100M website views per year

At BMC, research is always in progress.

Learn more biomedcentral.com/submissions 